\title{
Development of Android Controlled Arduino based Intelligent Car Parking System
}

\author{
Khalid Mahboob, Ibad Ur Rehman, Muhammad Ali, Mohammad Taha Khan, Muhammad \\ Rehman Nazir
}

Dept. of Software Engineering,Sir Syed University of Engineering \& Technology, Karachi 75300, Pakistan

Received: 02 November 2019; Accepted: 26 December 2019; Published: 08 February 2020

\begin{abstract}
The automobile industry is currently looking at the technology needed to move from today's original autonomous autos to a self-contained and safe driving solution. The automobile industry has been remarkably successful in producing reliable, safe, and affordable cars over the past century. Due to the significant progress made in computers and telecommunications, an autonomous car became a reality. In this regard, an android driver-less car is a vehicle that uses a combination of motors, software, and sensors to park cars between destinations without a human operator. To be fully autonomous, vehicles must be able to travel unmanned to a pre-determined destination on roads that are not fit for use. In this paper, the android controlled Arduino based intelligent car parking development stages and functionalities has been discussed. The motor system will be composed of the dc motors that run the car as well as the wheels and body of the car. The DC motor controls the circuit and a software driver. The android application will drive the car forward, reverse, left, and right (stopping will be the absence of a forward or backward command). It will do this by means of the software driver. There is also one motor which holds the brake and release. The significance of this system is that it has a distinctiveness to control real cars in real-time with android applications including steering control, gear shifting, horn control, and engine on/off. It has a self-parking system in a narrow crowded system through the sensors reading the environment and with actuators, a car could be park itself. Finally, on enabling effective automobile safety and efficient automotive cars, some of the challenges are needed to be addressed (and to provide) useful suggestions for approval by car manufacturers, designers, policymakers, and regulatory bodies.
\end{abstract}

Index Terms: Parking, car, Android, Bluetooth, motor

(C) 2020 Published by MECS Publisher. Selection and/or peer review under responsibility of the Research Association of Modern Education and Computer Science

\footnotetext{
* Corresponding author

E-mail address: nedian07@gmail.com
} 


\section{Introduction}

Auto parking has been an integral part of everyday life ever since. It is difficult to park the vehicle on your own. It's sometimes very difficult for the car drivers to accurately decide the vehicle surrounding the situation, especially for traditional vehicles, particularly within the rear of it. Additionally, parking of a car could be a complex operation for drivers among a really short time through the dominant steering wheel either to accelerate or apply the brake. A rise within the number of vehicles among a restricted and narrow parking space needs expert parking skills $[1,15]$.

Due to a large number of traffic accidents and driver requirements, automatic parking system focuses on the never-ending automotive engineering. Driving safety can be increased by automatic parking systems. It helps the driver to learn about the environment around the vehicle during the process of parking through different sensors [2,3]. Only the driver had to control the acceleration and brakes to park the car in a parking lot. In this way, it can reduce the weakness of drivers that will improve driving comfort. A large number of automobiles manufacturers and research institutions carry out research and development of intelligent car parking system [1].

In this regard, smartphones are becoming more and more powerful with an improved processor, greater storage capacity, more entertainment, and more communication functions today [2]. Smartphones have changed much of the normal mode of machine interaction. Android is an open-source platform widely used in smartphones in modern ages. Android is a complete software package containing the operating system, middleware layer, and large applications. Android smartphones are greatly advanced the commercial, industrial and applications for other general purposes [13].

In the same way, many technologies such as GSM, Wi-Fi, WLAN, and Bluetooth are used vastly today. Each technique has its own unique features and applications. Among these wireless connections, Bluetooth technology is typically implemented [5]. Bluetooth technology has changed the way people use digital devices at home or office and transported the traditional cabled digital devices into wireless devices [2, 4].

Also, DC motors employed in this study, are widely used to provide variable speed drive systems in industrial applications that resemble electrical automation, military equipment, high-capacity fixed drives, noise-free operation, compact operation, reliability and low maintenance and cost [2].

Here, the main research objective is to develop an Android application for controlling the car using Bluetooth. The hardware of the system consists of a controller supplied with the Bluetooth communication module. It will be connected to the DC motor and other additional components of the car. Once the car automation application is turned on and connected to the system via Bluetooth, one can control the vehicle by giving wireless commands from the program operation to the functions already in an application $[2,5]$.

Using ultrasonic sensors, an AI-based controller using the Arduino will control and park the car with ambient readings, so the AI controls steering, gears, accelerate and decelerate operations of the car during parking. This means that the AI-based controller will park the car straight and parallel mode according to the specified setting $[6,7]$.

The rest of the paper is organized as follows: Section II presents the Related Works, Section III describes the Experimental Setup with its implementation and working, Section IV consist of Results and Discussion which shows how the smart parking system is user efficient and beneficial, and Section V concludes the paper.

\section{Related Works}

Various techniques have been adopted for the development of an intelligent car parking system. Investigation of these systems indicates that they need more or fewer people interference for the process.

A study in [2] demonstrates a car controlled by an android application. The remote buttons are developed in the android application to control the motion of the car. Bluetooth communication is used for communication between car and smartphone applications. At most, the command is sent to the local Bluetooth module in a microcontroller using Bluetooth Input smartphones to control the motion of a car. 
In [3], an IoT based smart car parking system is presented. The system planned provides real-time information on parking availability in a parking space. Remote users can reserve parking spaces through the mobile applications. The entire system is centered on a wifi network. It saves time, fuel, and traffic congestion can be reduced.

Smart parking system based on a booking parking slot implemented in [4] using the Android application. PIC16F877A controller, IR sensor, RFID tag, Relay, LED and Android-based mobile used to perform an experiment. Cost is calculated for a slot automatically when the process is done using the RFID tag.

A new smart parking system is proposed in [5] for an urban environment. An optimum car parking space is assigned and reserved by the system for the driver depending on the user's needs that combine parking destination and cost and also ensure effective utilization of overall parking capacity. The Mixed Integer Linear Program (MILP) problem is solved by the approach at each decision point in time sequence. The solution to any MILP is the optimal allocation based on the current status and subject of random events, such as new user requests or available parking spaces.

A prototype of the Smart Parking System on the basis of reservation (SPSR) is designed and implemented in [6] to allow drivers to find and book empty parking spaces. A parking reservation policy implemented in this system to balance the advantage of service providers and user requirements. The proposed system can reduce traffic congestion and traffic volume seeking vacant parking slots.

Automated car parking system architecture is proposed in [7] controlled by the Android application. The proposed system uses an Android application to facilitate car parking and retrieval of the car to the customer. The proposed design provides an effective parking system using an effective search method supported by effective processing of the GSM module, RF module, and microcontroller.

In [8], a system for car parking is detected by the sensors and the analog signals are operated in the Arduino. At most, details about slots such as slot numbers and whether free or parked slots are provided to users as text. Slots are refreshed every 3 seconds. The first step is sensing the parking slot through the sensors. The second step is information detection processed in Arduino. The third step is that users can receive parking details on their smartphones via GPS to allocate the slots they need.

Intelligent Parking System (IPS) is used in [9] to book parking space using the Android device. Users can check the status of the parking slot and reserve the parking slot in advance. Each time a user reserves a specific slot highlight in red and all vacant slots will be green. Reservations can be made via credit/internet banking. This application also offers additional functionality to cancel the slot within 20 minutes of booking.

The aim of the study in [11] is to automate the car using the android application. It discusses the project which presents a model of a car parking system that can regulate and manage the number of cars that can be parked in a space at any time based on the availability of parking spot. Android parking is a method of parking cars using sensing devices. The entering from the parking lot is commanded by an Android-based application.

An investigation in [12] focuses on the situation of chaotic traffic with not enough vehicle parking area. People keep on roaming on roads searching for a parking space to park their vehicles especially at peak hours of time. The system proposed a smart parking system that regulates a number of vehicles to the nearest parking space at any given time based on the parking space availability.

\section{Experimental Setup}

An android controlled Arduino based intelligent car parking system is developed using different components with various features. The hardware components consist of Bluetooth HC-05 (Master and Slave), 3 DC Stepper Motors (Vertical, Horizontal, and Steering), Microcontroller (Arduino Mega), Motor Driver (tb6600), Ultrasonic sensor HRC-S04, and Smartphone (Android 4.0+). The software components used in an experiment are Android Studio, C programming, Java, and Arduino integrated development environment (IDE) [13, 14].

\subsection{Ultrasonic Sensors:}


Fig. 1. depicts the position of ultrasonic sensors that work by emitting sounds that are higher pitched than the human ear can easily detect $[1,8]$. These sound waves bounce off nearby objects, sending the signals back to the sensor. The sensor is able to create an approximate image of the surrounding area by measuring how long it takes for sound waves to bounce back and gives the signal $[5,6]$.

In this figure, there are 10 sensors in which 3 are located in the front, 2 at left, 2 at right and 3 at the backside of the car. These sensors are ultrasonic parking sensors which sense the environment by sounds that are higher pitched.

The front 3 sensors are located at the front bumper which senses the left, right and middle of the front side and gives output to the connected device. The left-front-sensor, right-front-sensor and front sensor named respectively. Left-front-sensor calculates the distance and makes sure to program whether distance available or not. The right-front-sensor checks the distance of the right front while the front sensor has the same function.

The left 2 sensors are located at left two doors of the car which senses the environment of the left sides of the car. This sensor specially located there for vertical and parallel parking to the left side. Firstly, it will measure left side distance if space available for parking and give a signal to the program in order to proceed to the next step.

The right 2 sensors are located at the right two doors of the car which senses the environment of the right sides of the car. They also have the same function as the left sensor but for the right side. The back 3 sensors are located rear bumper of the car which senses left, the right, and the middle environment of the back sides of the car. They are specially located there to finish the parking proceed. These sensors read back-side distance and stop the car in a secure distance. 10 sensors are located especially for the security purpose of the car so that there will minimum chance of any damages.

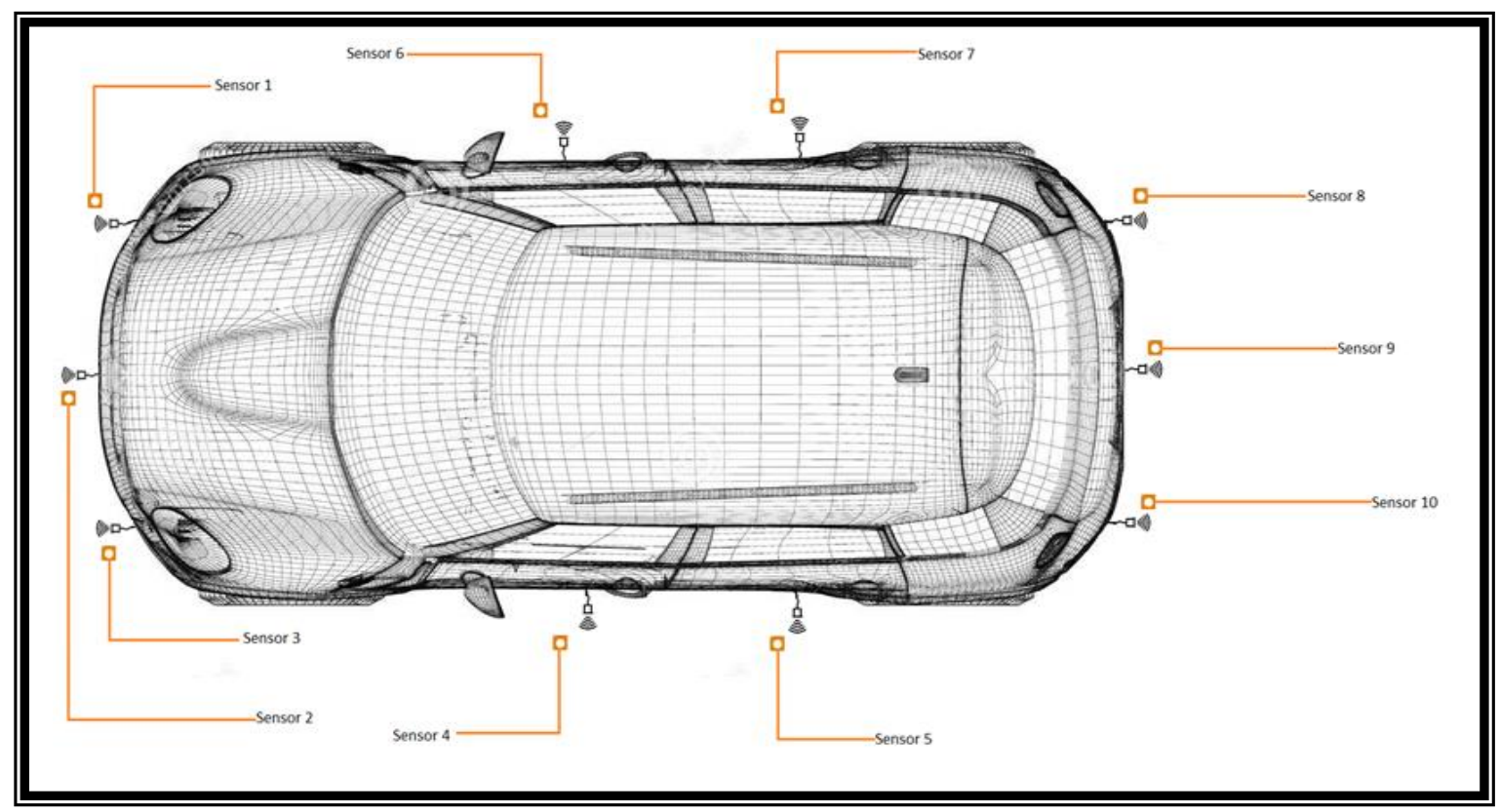

Fig.1. Position of Ultrasonic Sensors

\subsection{Steering Function:}

The gear is put to the steering rod by cutting the gear from middle and two nut bolts are welded, one at the corner and second nut bolt at the opposite side. Firstly, it takes the diameter of the steering rod and makes 
accurate measurements, after that, it fix the gear and then tightens the nut bolt at both sides with the help of a spanner and fixes it perfectly until it is not able to slip or move.

Secondly, after fixing the gear to the rod a foundation is made by taking the diameter of space behind the steering rod and fix the DC motor namely NEMA 23, which operates the gear and leads to move the steering. This gear is easy to remove by just opening the nut and bolt from both sides and the gear is out.

The DC motor foundation can also be fixed easily which depends on the nut bolt, however, the DC motor foundation can be removed by removing the nut bolt and it is out of that specific space [2].

\subsection{Stepper Motor:}

A stepper motor is an electric motor used to convert electrical power into mechanical power. Also, it is a synchronous electric motor that can divide a full rotation into the required number of steps. The motor's position can be controlled without any feedback, as long as the motor is carefully programmed according to the application. Stepper Motor handles the car speed by counting the steps. The accurate results are found through this motor using Arduino based microcontroller which sends the command to the motor to perform what is required. Two Stepper Motors namely NEMA23 Bipolar Stepper Motor and High Torque NEMA 23 CNC Stepper Motor have been used in developing this intelligent car parking system shown in Fig. 2 and 3.

Fig. 2. NEMA 23 Stepper Motor Bipolar
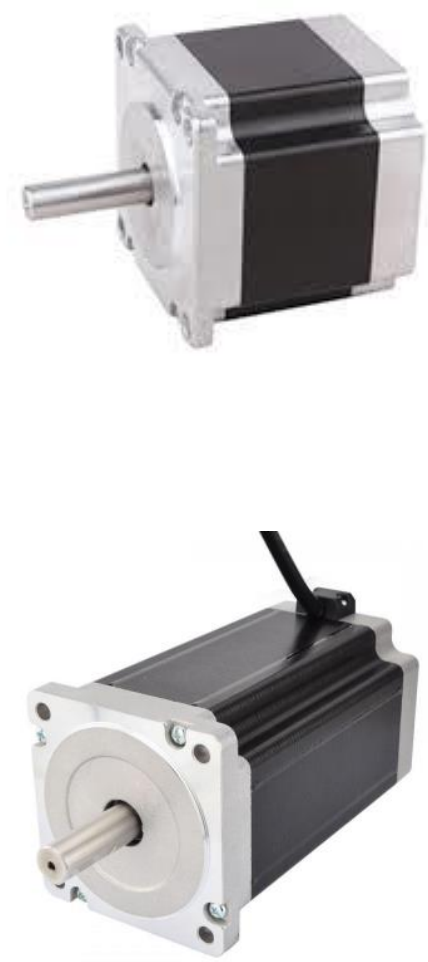

Fig. 3. High Torque NEMA 23 CNC Stepper Motor 


\subsection{Arduino based Circuit:}

The sensor and motors are directly connected to Arduino while Arduino connected to mobile through Bluetooth displayed in Fig. 4. When the user hits any command through mobile, it will be received by Arduino and Arduino control the circuit $[8,14]$.

The wires are placed at different positions to let the motor work perfectly. First, the accurate combinations of wires are acquired and then the commands are sent to Arduino which gets the code and gives commands to Stepper Motor that how and when to work at which timing.

Many experiments have been performed on the circuit to get perfect combinations of wires and perfect points at which the motor runs perfectly.

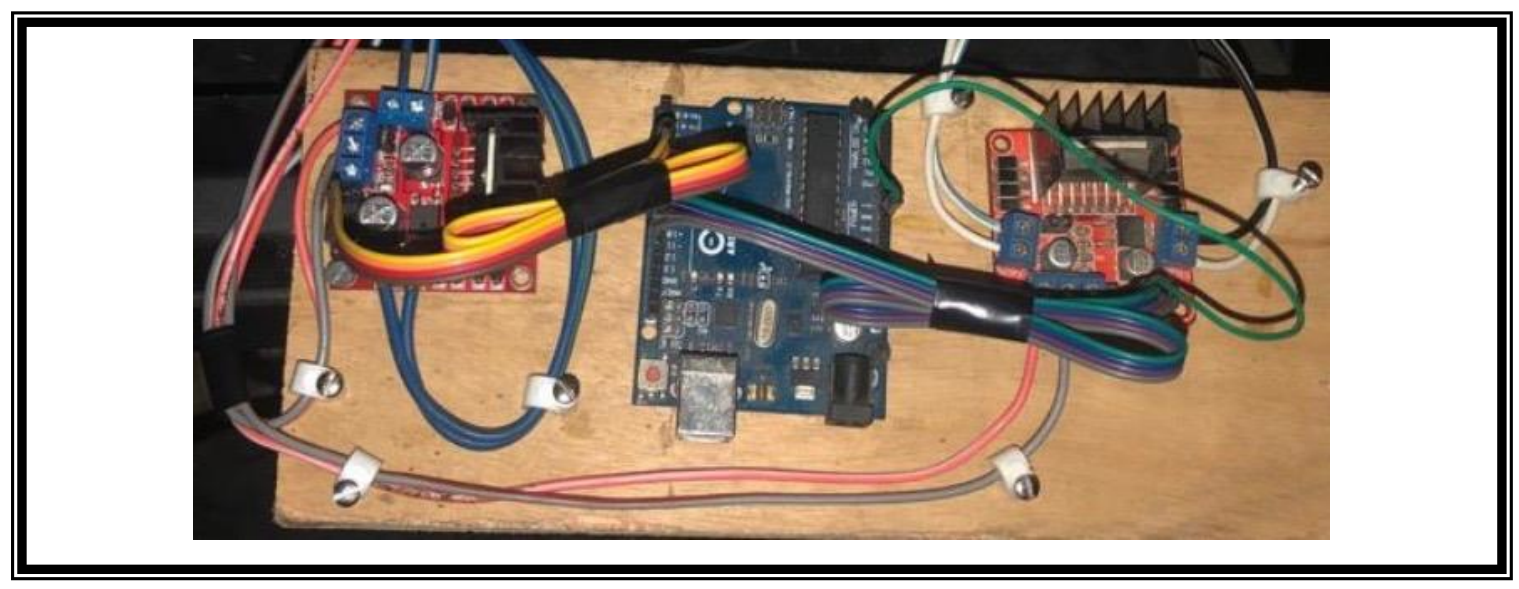

Fig. 4. Arduino based circuit through Bluetooth

\subsection{Shaft for Gear:}

The shaft specially designed for the car gear movement as shown in Fig. 5. This shaft works by moving forward, backward, left and right. The Stepper Motor gets the command through the Arduino (code) and works at the desired commands in which the motor use to change gear shifting of the car by motor.

This shaft is operated by two motors in which one motor is able to move the shaft left and right, and one motor is able to move the shaft forward and backward [2].

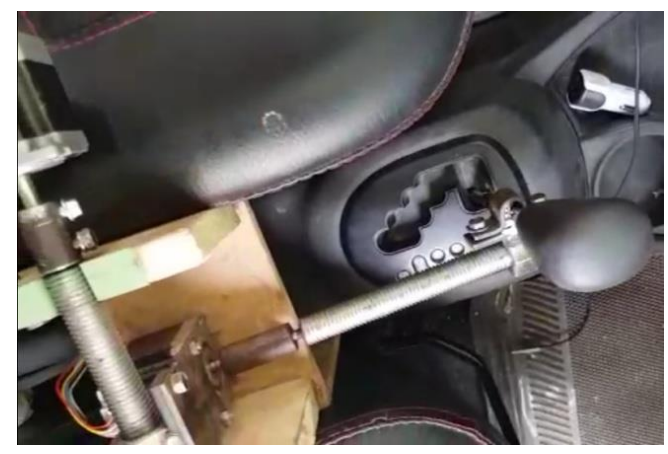

Fig. 5. Shaft for gear movement 


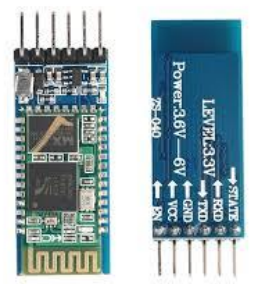

Fig. 6. Bluetooth Module HC-05

\subsection{Bluetooth Module:}

The HC-05 module is an easy-to-use Bluetooth SPP (Serial Port Protocol) module, specifically designed for the transparent setup of wireless serial connections illustrated in Fig. 6. Bluetooth serial port module is completely authorized Bluetooth V2.0 + EDR (Enhanced Data Rate) 3Mbps module with a full $2.4 \mathrm{GHz}$ radio transceiver and baseband. CSR Bluecore 04 which is an external single chip is used with AFH (Adaptive Frequency Hopping Feature) and with CMOS technology. It has a small footprint of $12.7 \mathrm{~mm}$ by $27 \mathrm{~mm}$ [2].

\subsection{Android User Interface:}

The android based user interface is designed simply as much as possible so that the user can use it easily [3]. Firstly, the paired device connection page will appear using the android application menu [13] as shown in Fig. 7. It requires connecting to the Arduino only when the Bluetooth is activated. On a successful connection, the two modes of parking are available in an application either through mobile Manual mode as illustrated in Fig. 9 or AI mode as displayed in Fig. 10. The purpose of both modes is described below:

\subsubsection{Manual Mode:}

In manual mode, the left and right buttons are used for steering control. It will rotate steering in a clock-wise and anti-clockwise direction. Button stop will apply the brake at any position while the forward button provides acceleration to the car and a backward button is used for car reverse operation.

\subsubsection{AI Mode:}

The AI has two modes of parking: straight and parallel for the automatic front and backward parking accordingly. If the failure occurs using an AI mode for any reason, the car will beep five times and will stop.

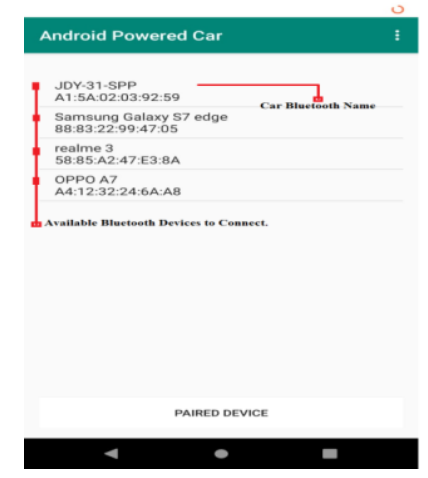

Fig. 7. Pairing with Bluetooth 


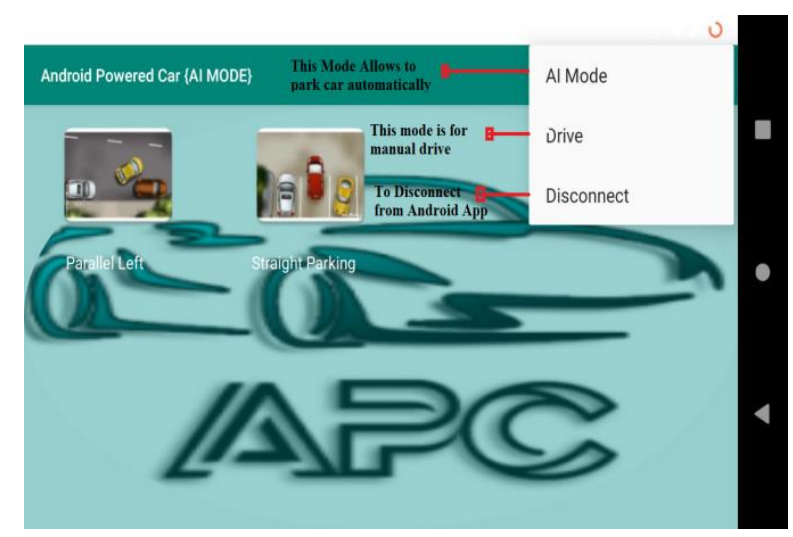

Fig. 8. Displaying options for parking

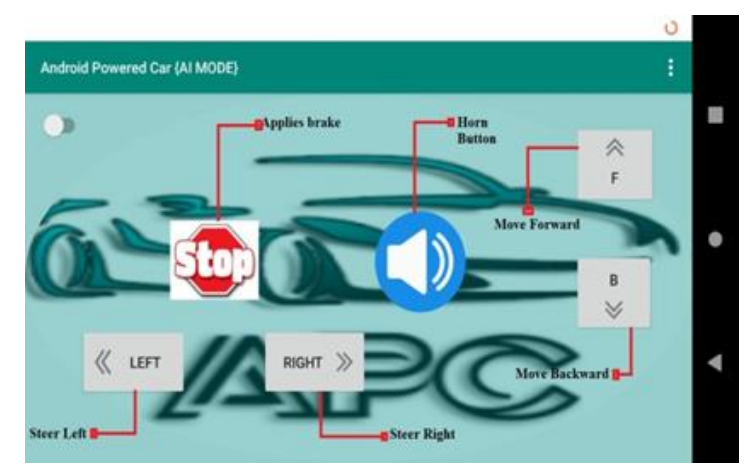

Fig. 9. Manual Mode

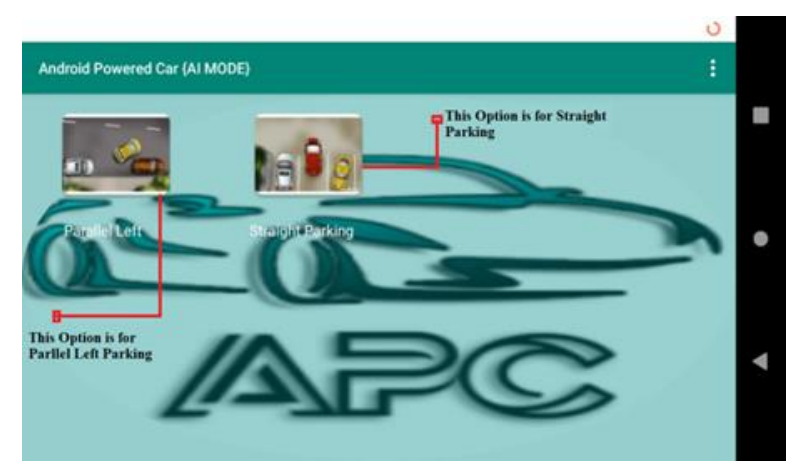

Fig. 10. AI Mode

In summary, the car starts with pairing Bluetooth. In Fig. 7, the application shows all the pairing devices. The user has to connect his android phone with the car Bluetooth module. Once the application connects with Bluetooth, it proceeds to drive mode (Manual) $[8,13]$. According to the given interface user can start the car with a switch button, he can steer the steering in either direction with the left or right button. He can stop the car with the stop button and beep the horn with the horn button manually as shown in Fig. 9. If the user wants to move the car in AI mode, he can select AI mode with the given options as shown in Fig. 8. The user can 
select two modes of parking in AI mode i.e., straight parking and parallel parking. Again, if the user wants to move the car using drive mode (Manual), he can select it with the given options as shown in Fig. 8. After performing the parking operations the user can use the disconnect option [15].

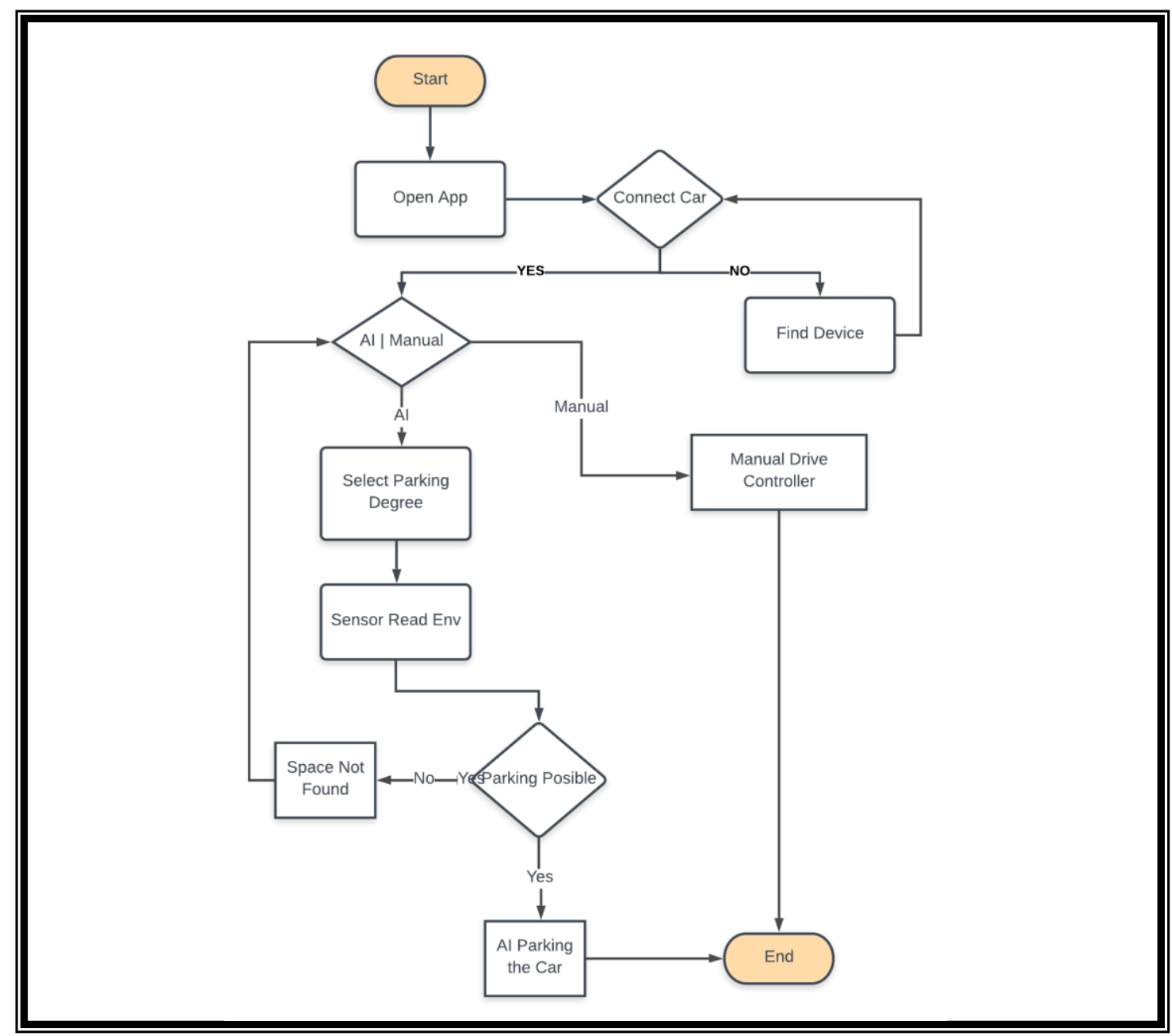

Fig. 11. Flow of Intelligent car parking system using an Android application

The flow of the overall intelligent car parking system using the Android application is shown in Fig. 11. The overall system starts with an android application to control the car through mobile [9]. Initially, the user has to connect his mobile with the system via Bluetooth. After that, the user can see two modes of parking with mobile: Manual mode and AI mode. If the user wants to choose the manual mode with mobile, he can use the given interface else he can choose AI mode from the menu. Using AI a user can choose parking modes: straight or parallel. After sending command, the controller system reads the environment and if space is available, sensor command to the program proceeding further steps. Otherwise, the car will not move from the specified position [4]. 


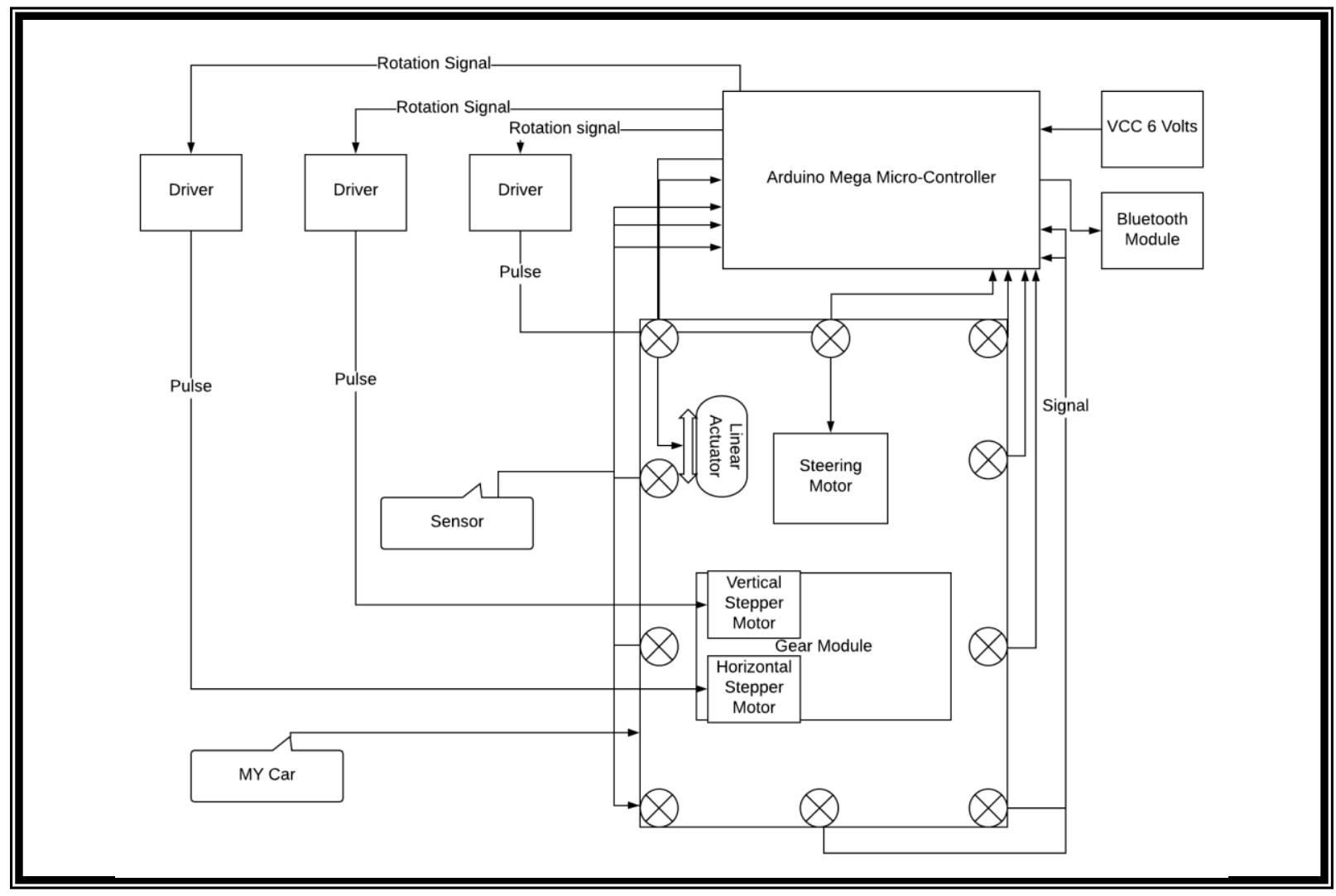

Fig. 12. Block Diagram of the System

Fig. 12. shows the overall block circuit diagram of the hardware which shows the connections between Arduino, Bluetooth, motors, and sensors. It also shows the three Stepper Motor drivers TB-6560 which is responsible for the movement of the motors in either clockwise direction or anti-clockwise direction. The two motor drivers are connected with gear motors and one is connected with the steering to rotate it in either direction. The supply of 6volts is provided to the motors. RxD pin of the Arduino is connected to the TxD pin of Bluetooth and vice versa [14]. Three sensors are connected in front of the car and three sensors are on the back. While two sensors are on the right and left of the car. Sensors are directly connected with Arduino with a supply of $5 \mathrm{~V}$.

\section{Experimental Results}

The system tests are done in various environments using a specific path. The sources are ordinary (or virtual) own created environment. The results achieved from the tests involve four aspects as follows:

- The connectivity of mobile android application and microcontroller via Bluetooth is tested to determine whether it can adjust the microcontroller method for the motor or not.

- The acceleration, steering and overall system are tested by commanding via mobile to ensure all functionality is working fine. 
- The sensors are tested to determine whether it can collect the path information accurately or not by observing the commands from the microcontroller.

- The smart car steering is tested for whether it is sensitive and smooth when the car runs on the tracing path.

Through the microcontroller, a user can command the path information through the mobile by the Bluetooth module. Thus, the smart car can collect and extract the path information accurately [7].

The overall circuit is tested by sending a command from the mobile. As a result, there is no collision between signals, therefore, all the motors and sensors working according to the given environment and scenario.

The android application is designed to connect with Bluetooth (HC-05) only [2]. The system is checked to connect the android application with other Bluetooth devices but it denies pairing with other devices rather than Bluetooth (HC-05) which means system working correctly [10].

\section{Conclusion}

The intelligent car parking system based on Arduino is implemented using the Android application. There seem a lot of potentials required for autonomous parking for this study. But the strategic execution of this system lies in the safe implementation of this technology and safe implementation can only be done by applying stringent rules and standards. So, by developing the intelligent car parking system, the car can be controlled with the help of Android application in smartphones and Ardunio based microcontroller in a car. Thus reducing the effort of controlling the car in rough terrain and it also helps in reducing the difficulties of parking a car in narrow parking conditions.

The communication between the car and smartphone has been done with the help of Bluetooth communication. The commands are transmitted to the Bluetooth module in the microcontroller using Bluetooth in smartphones. This paper advances the overall design goals and structure of a brief introduction at first. Then according to the requirements of the entire system, the system is divided into different functional modules. Each module's design and implementation, as well as intelligent control ideas in each module, are described in detail.

At last, the final system has been tested using functional tests. Through the tests' results, it has been determined that the intelligent car parking could be controlled through the Android mobile manual and AI parking modes. It shows that intelligence control research based on the smart car is successful. There will be broader application prospects that combining intelligence control technology and embedded technology. It will promote great economic benefits to the growth of the automobile industry.

\section{Limitations and Future Directions}

It is found that there are still some gaps that need to be considered more deeply. These gaps suggest new directions for future research. The most promising directions are mentioned here:

- Performances of sensors are not very stable. If the weather is bad, such as rain and snow, the effects of sensors are greatly decreased. Sensors are also not very smart. For example, when a car encounters an object, the sensors regard it as an immovable obstruction and guide the car to change the parking path. In fact, the object will automatically leave when the car is closer to it. So finding a kind of stable and smart sensor is still an important issue.

- The current automatic parking system cannot be considered as complete automatic parking in all cases. Parking spaces have size limitations and a vehicle cannot achieve its automatic diagonal parking. The automatic parking can only be done on some ideal parking spaces and this undoubtedly gives an important research direction. 
- System disturbances and environment uncertainties cannot be avoided. For example, because a vehicle often slides on muddy roads, the vehicle dynamics characters cannot be determined accurately.

- With an increase in electronics and computerization of the vehicles there are more concerns about cyberattacks. There should be proper fail-safe plans if the vehicles are lost from the grid or connection is lost with the controller.

\section{Acknowledgements}

This research was funded by IGNITE. We thank our colleagues from Sir Syed University of Engineering \& Technology who provided insight and expertise that greatly assisted the research.

\section{References}

[1] Wang, W., Song, Y., Zhang, J., \& Deng, H. (2014). Automatic parking of vehicles: A review of literatures. International Journal of Automotive Technology, 15(6), 967-978. doi:10.1007/s12239-014-0102-y.

[2] M. J.G., P. P. A.S., Joseph, D., P. S. Android controlled Smart Car. International Journal of Computer Engineering In Research Trends. 2015Dec; 2(12):1087-90.

[3] Bachhav, J.D., A. M.M. Smart Car Parking System. International Research Journal of Engineering and Technology (IRJET) . 2017Jun; 4(6):3036-8.

[4] R. R., Dhanalakshmi S. Android based smart parking system using slot allocation \& reservations. ARPN Journal of Engineering and Applied Sciences. 2015Apr;10(7):3116-20.

[5] Geng, Y., \& Cassandras, C. G. (2012). A new "Smart Parking" System Infrastructure and Implementation. Procedia - Social and Behavioral Sciences, 54, 1278-1287. doi:10.1016/j.sbspro.2012.09.842.

[6] Patil, M., Sakore, R. Smart Parking System Based On Reservation. International Journal of Scientific Engineering and Research (IJSER). 2014 Jun; 2(6):21-6.

[7] Bonde, D. J., Shende, R. S., Kedari, A. S., Gaikwad, K. S., \& Bhokre, A. U. (2014). Automated car parking system commanded by Android application. 2014 International Conference on Computer Communication and Informatics. doi:10.1109/iccci.2014.6921729

[8] Kiruthika, S., Surendran, D. Smart Car Parking using Arduino and Android Application. International Journal of Computer Science and Mobile Computing (IJCSMC). 2016Feb; 5(2):230-4.

[9] Anitha, J,, Thoyajakshi, Y., Ramya, A., Sravani, V., Kumar, P. Intelligent Parking System Using Android Application. International Journal of Pure and Applied Mathematics. 2017Apr14; 114(7):165-74.

[10] Mitra A, Patel KD, K D. Android Based Smart Parking Reservation. International Journal of Innovative Research in Computer and Communication Engineering (IJIRCCE). 2016Sep; 4(9):15845-9.

[11] Moore, M.M., Lu, B. Autonomous Vehicles for Personal Transport: A Technology Assessment. SSRN Electronic Journal. 2011. doi: 10.2139/ssrn.1865047.

[12] Frazzoli E, Dahleh M, Feron E. Real-time motion planning for agile autonomous vehicles. Proceedings of the 2001 American Control Conference (Cat No01CH37148). 2001. doi: 10.1109/acc.2001.945511.

[13] https://www.android.com/ [cited 2019 July 25].

[14] https://www.arduino.cc/ [cited 2019 August 05].

[15] https://www.ll.mit.edu/publications/labnotes/automation/ [cited 2019 August 15]. 


\section{Authors' Profiles}

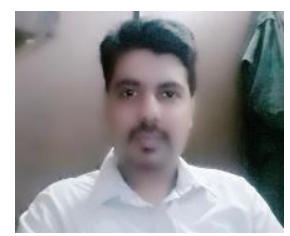

Khalid Mahboob earned master's degree in Computer Science \& I.T. in 2011 from NED University of Engineering \& Technology, Karachi, Pakistan. Currently, he is a Ph.D Scholar at NED University of Engineering \& Technology, Karachi, Pakistan. He is a Lecturer in Software Engineering Department at Sir Syed University of Engineering \& Technology, Karachi, Pakistan. His research interests include, Educational Data Mining, Machine Learning, Expert System and Decision Support System, Sentiment Analysis, Computer Programming, Pedagogics.

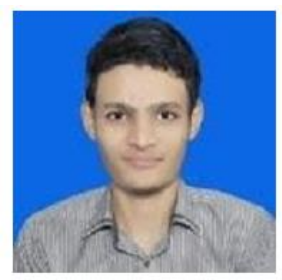

Ibad Ur Rehman is an under-graduate student at Software Engineering Department of Sir Syed University of Engineering \& Technology, Karachi, Pakistan. He is a Junior Software Engineer at Technology Ally. His research interests include, Artificial Intelligence, Circuit Designing, System Programming.

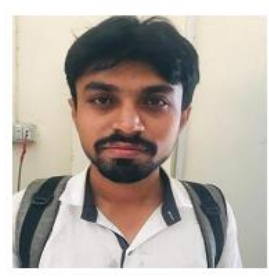

Muhammad Ali is an under-graduate student at Software Engineering Department of Sir Syed University of Engineering \& Technology, Karachi, Pakistan. His research interests include, Software Development, Software Quality Analysis, Artificial Intelligence, Android Programming.

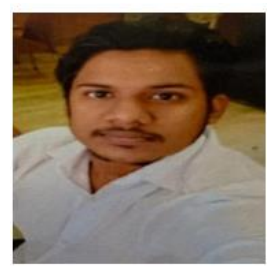

Mohammad Taha Khan is an under-graduate student at Software Engineering Department of Sir Syed University of Engineering \& Technology, Karachi, Pakistan. His research interests include Artificial Intelligent System, Desktop Computing, Wireless and Mobile Computing, Sensors.

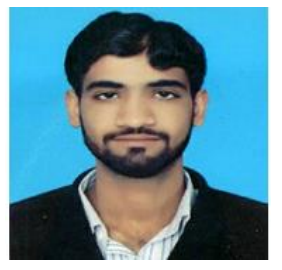

Rehman Nazir is an under-graduate student at Software Engineering Department of Sir Syed University of Engineering \& Technology, Karachi, Pakistan. His research interests include Software Development, Object Oriented Designing, Circuit Design and Applications, Intelligent Automation and Control. 
How to cite this paper: Khalid Mahboob, Ibad Ur Rehman, Muhammad Ali, Mohammad Taha Khan, Muhammad Rehman Nazir, " Development of Android Controlled Arduino based Intelligent Car Parking System ", International Journal of Wireless and Microwave Technologies(IJWMT), Vol.10, No.1, pp. 48-61, 2020.DOI: $10.5815 / \mathrm{ijwmt} .2020 .01 .05$ 